\title{
THE DUAL TOPOLOGY FOR THE PRINCIPAL AND DISCRETE SERIES ON SEMISIMPLE GROUPS( $\left.{ }^{1}\right)$
}

\author{
BY \\ RONALD L. LIPSMAN
}

\begin{abstract}
For a locally compact group $G$, the dual space $\hat{G}$ is the set of unitary equivalence classes of irreducible unitary representations equipped with the hullkernel topology. We prove three results about $\hat{G}$ in the case that $G$ is a semisimple Lie group: (1) the irreducible principal series forms a Hausdorff subspace of $\hat{G}$; (2) the "discrete series" of square-integrable representations does in fact inherit the discrete topology from $\hat{G} ;(3)$ the topology of the reduced dual $\hat{G}_{r}$, that is the support of the Plancherel measure, is computed explicitly for split-rank 1 groups.
\end{abstract}

1. Introduction. It has been a decade since Fell introduced the hull-kernel topology on the dual space $\hat{G}$ of a locally compact group $G$. Although a great deal has been learned about the set $\hat{G}$ in many cases, little or no work has been done relating these discoveries to the topology. Recent work of Kazdan indicates that this is an unfortunate oversight. In this paper, we shall give some results for the case of a semisimple Lie group.

Let $G$ be a connected semisimple Lie group with finite center. Associated with a minimal parabolic subgroup of $G$ there is a family of representations called the principal series. Our first result states roughly that if we strike out those members of the principal series that are not irreducible (a "thin" collection, actually), then what remains is a Hausdorff subspace of $\hat{G}$. We shall determine the topology precisely.

Next, suppose $G$ has a compact Cartan subgroup. Then it is known that $\hat{G}$ contains a family $\mathscr{D}$ of square-integrable representations called the discrete series. We shall justify this name by showing that the hull-kernel topology restricted to $\mathscr{D}$ is indeed discrete.

Finally, in the special case of split-rank 1 groups, we shall determine completely the topology of $\hat{G}_{r}$, the smallest closed subset of $\hat{G}$ which supports the Plancherel measure. We will verify a conjecture of Dixmier in this case.

The representations of $G$ induced from nonminimal parabolic subgroups are

Received by the editors August 24, 1969 and, in revised form, February 3, 1970.

AMS 1968 subject classifications. Primary 2250, 2260; Secondary 5420.

Key words and phrases. Semisimple Lie groups, irreducible unitary representations, dual space, hull-kernel topology, principal series, discrete series, Plancherel measure, universal enveloping algebra, characters, invariant eigendistributions, Cartan subgroups, root systems, Weyl group.

(1) The preparation of this paper was partially supported by the NSF through GP-13871. Copyright (C) 1970, American Mathematical Society 
being studied by Professor Harish-Chandra. It seems quite likely that, given the character of these representations and results on irreducibility analogous to Bruhat's for the principal series, our techniques will be applicable to these series.

Many people have conjectured that if $G$ has a complex structure then all the representations of the principal series are irreducible (as is the case for $\operatorname{SL}(n, C))\left({ }^{2}\right)$. It would follow immediately from our work that $\hat{G}_{r}$ is Hausdorff (thus substantiating a conjecture transmitted to me by Professor Fell in private correspondence).

I would like to express here my deep gratitude to Professor R. Langlands for many valuable conversations. Originally, my work on the principal series was restricted to the complex semisimple case. Langlands encouraged me to expand the results in order to include real groups. Among other helpful suggestions, I would like to cite two specifically:

(1) He pointed out the necessity of reconciling the Weyl groups of Bruhat and Harish-Chandra (see Lemma 4.2). The relevance of equation (4.3) was observed by him also, although the proof is my own.

(2) My original approach to Lemma 8.2 was via global characters. The proof was quite cumbersome and not nearly as general as the one appearing here. The method of infinitesimal characters was suggested by Langlands.

In a preliminary version of this paper I restricted myself to groups having a faithful matrix representation. I would like to thank the referee for drawing my attention to Satake's paper [6]. By using a result in [6] and several pertinent suggestions from the referee, I was able to drop this (and another technical) assumption. In this regard see Lemma 3.1 and the first paragraph of the proof of Theorem 6.2.

Notation. Let $G$ be a Lie group with Lie algebra $\mathfrak{g}$. If $\mathfrak{h} \subseteq \mathfrak{g}, A \subseteq G$ are subsets and $x \in G$, we denote by $\mathfrak{h}^{x}$ and $A^{x}$, the sets $\mathfrak{h}^{x}=\{\operatorname{Ad} x(H): H \in \mathfrak{h}\}, A^{x}=\left\{x a x^{-1}: a \in A\right\}$. More generally we let $\mathfrak{h}^{G}=\bigcup_{x \in G} \mathfrak{h}^{x}$ and $A^{G}=\bigcup_{x \in G} A^{x}$. We also systematically use

$$
\begin{array}{ll}
Z(\mathfrak{h})=\{x \in G: \text { Ad } x(H)=H, \text { all } H \in \mathfrak{h}\}, & N(\mathfrak{h})=\left\{x \in G: \mathfrak{h}^{x} \subseteq \mathfrak{h}\right\}, \\
Z(A)=\{x \in G: x a=a x, \text { all } a \in A\}, & N(A)=\left\{x \in G: A^{x} \subseteq A\right\} .
\end{array}
$$

$C^{\infty}(G)$ and $C_{0}(G)$ will denote the infinitely differentiable functions and continuous functions of compact support, respectively. As usual, $C_{0}^{\infty}(G)=C^{\infty}(G) \cap C_{0}(G)$. Finally, we use $\|\cdot\|_{\infty}$ to denote the uniform norm for operators on Hilbert space.

2. The dual topology. Let $\mathbb{E}$ be a $C^{*}$-algebra, $T$ a $*$-representation of $\mathfrak{C}$, and $\mathscr{S}$ a family of *-representations of $\mathbb{E}$ (all representations act in Hilbert space). We say $T$ is weakly contained in $\mathscr{S}$ if $\operatorname{Ker}(T) \supseteq \bigcap_{S \in \mathscr{S}} \operatorname{Ker}(S)$. Let $\hat{\mathfrak{E}}=$ the unitary equivalence classes of topologically irreducible *-representations of $\mathfrak{夭}$. Give $\hat{\mathfrak{C}}$ the topology determined by making weak containment a closure operation. This is the hullkernel topology $[3, \S 4]$.

$\left.{ }^{2}\right)$ This has been proven at last; see N. Wallach, The irreducibility of the full principal series for complex semisimple Lie groups (to appear). 
Next let $G$ be a locally compact group and $C^{*}(G)$ its associated group $C^{*}$ algebra $[3, \S 3]$. It is well known that there exists a $1-1$ correspondence between $C^{*}(G)^{\wedge}$ and $\hat{G}=$ the unitary equivalence classes of irreducible unitary representations of $G$. $\hat{G}$ with the hull-kernel topology transferred from $C^{*}(G)^{\wedge}$ is called the dual space. See [3, Theorem 1.5] for equivalent conditions on the topology involving uniform-on-compacta limits of positive definite functions.

Define the reduced dual $\hat{G}_{r}$ to consist of those members of $\hat{G}$ which are weakly contained in the regular representation. If $G$ is separable, unimodular and type I (e.g. a connected semisimple Lie group), then $\hat{G}$ has a unique Plancherel measure (see $[2,18.8 .2])$. It follows from $[2,18.8 .4]$ that $\hat{G}_{r}$ coincides with the support of the Plancherel measure.

We now restate two results from [3] which will be fundamental to our work. If $\mathfrak{C}$ is a $C^{*}$-algebra and $\mathscr{S}$ is a family of *-representations of $\mathfrak{E}$, then $f \in \mathfrak{C}$ is called boundedly represented in $\mathscr{S}$ whenever there exists $n$ such that $\operatorname{dim}$ range $T_{f} \leqq n$, all $T \in \mathscr{S}$.

Lemma $2.1[3$, p. 391]. Assume $\mathfrak{E}$ has a dense selfadjoint subalgebra $\mathfrak{A}$ such that every $f \in \mathfrak{A}$ is boundedly represented in $\hat{\mathfrak{C}}$. Let $\left\{T^{i}\right\}$ be a net of elements in $\hat{\mathfrak{E}}$ and $S^{1}, \ldots, S^{r}$ a finite sequence of elements in $\hat{\mathbb{E}}$ (not necessarily distinct) such that

$$
\lim _{i} \operatorname{Tr}\left(T_{f}^{i}\right)=\sum_{k=1}^{r} \operatorname{Tr}\left(S_{f}^{k}\right), \quad \text { all } f \in \mathfrak{A} .
$$

Then for all $S \in \hat{\mathfrak{E}}$, we have $T^{i} \rightarrow S$ (in the hull-kernel topology) $\Leftrightarrow S=$ some $S^{k}$, $k=1, \ldots, r$.

Lemma 2.2 [3, p. 392]. Let $G$ be a connected semisimple Lie group with finite center. Then $C^{*}(G)$ has a dense selfadjoint subalgebra $\mathfrak{A}$ with each element boundedly represented in $C^{*}(G)^{\wedge}=\hat{G}$.

We shall have occasion to examine $\mathfrak{A}$ more closely in $§ 6$.

3. Semisimple groups. Let $G$ be a connected, noncompact semisimple Lie group with finite center and $g$ its real Lie algebra. Fix a Cartan decomposition $\mathfrak{g}=\mathfrak{f}+\mathfrak{p}$ and let $K=$ the maximal compact subgroup of $G$ having $\mathfrak{f}$ as Lie algebra. Let $\mathfrak{h}_{+}$be a maximal abelian subalgebra of $\mathfrak{p}$. Extend $\mathfrak{h}_{+}$to $\mathfrak{h}$, a maximal abelian subalgebra of $\mathfrak{g}$. Then $\mathfrak{h}$ is a Cartan subalgebra of $\mathfrak{g}$ and if $\mathfrak{h}_{-}=\mathfrak{h} \cap \mathfrak{f}$, we have a direct sum decomposition $\mathfrak{h}=\mathfrak{h}_{-} \oplus \mathfrak{h}_{+}$.

Let $\mathfrak{g}_{c}$ be the complexification of $\mathfrak{g}$. Then the complexification $\mathfrak{h}_{c}$ of $\mathfrak{h}$ is a Cartan subalgebra of $\mathfrak{g}_{c}$. Consider the roots of $\left(\mathfrak{g}_{c}, \mathfrak{h}_{c}\right)$, i.e. the nonzero linear functionals $\alpha$ on $\mathfrak{h}_{c}$ such that $\mathfrak{g}_{\alpha}=\left\{X \in \mathfrak{g}_{c}:[H, X]=\alpha(H) X\right.$, all $\left.H \in \mathfrak{h}_{c}\right\} \neq\{0\}$. (Note. For any pair $(\mathfrak{g}, \mathfrak{h})$ consisting of a reductive Lie algebra and a Cartan subalgebra, when we speak of the roots of $(\mathfrak{g}, \mathfrak{h})$ we mean the roots of $\left(\mathfrak{g}_{c}, \mathfrak{h}_{c}\right)$. In addition, the Weyl group $W(\mathfrak{g}, \mathfrak{h})$ will be the group generated by the reflections of $\mathfrak{h}_{c}$ corresponding to a simple root system.) Let $P$ denote the positive roots of $(g, \mathfrak{h})$ with respect to some 
ordering. Define the subsets $P_{+}, P_{-}$as in [4a, p. 188]; roughly, $P_{-}$consists of those roots that vanish on $\mathfrak{h}_{+}, P_{+}=P-P_{-}$.

Let $\mathfrak{m}=$ the centralizer of $\mathfrak{h}_{+}$in $\mathfrak{t}$ and let $A^{\circ}, A_{-}^{\circ}, A_{+}, M^{\circ}$ be the connected Lie subgroups of $G$ having Lie algebras $\mathfrak{h}, \mathfrak{h}_{-}, \mathfrak{h}_{+}, \mathfrak{m}$, respectively. $A_{+}$is a vector group and $A^{\circ}$ is a maximal connected abelian subgroup of $G$. Let $A, A_{-}, M$ be the centralizers of $A^{\circ}$ in $G, A^{\circ}$ in $K$, and $A_{+}$in $K$, respectively. Then

$$
\begin{aligned}
A & =Z\left(A^{\circ}\right)=Z(\mathfrak{h}), \\
A_{-} & =Z\left(A^{\circ}\right) \cap K=Z(\mathfrak{h}) \cap K, \\
M & =Z\left(A_{+}\right) \cap K=Z\left(\mathfrak{h}_{+}\right) \cap K,
\end{aligned}
$$

and these are closed subgroups of $G$. Since $A^{\circ}, A_{-}^{\circ}, M^{\circ}$ are the respective connected components of the identity, they are closed also. We have $A^{\circ}=A_{-}^{\circ} A_{+}$; and $A=A_{-} A_{+}$is a Cartan subgroup of $G$ which is not necessarily abelian [4f, p. 556]. Finally, if $\mathfrak{n}_{c}=\sum_{\alpha \in P_{+}} \mathfrak{g}_{\alpha}$ and $\mathfrak{n}=\mathfrak{g} \cap \mathfrak{n}_{c}$, then the corresponding connected Lie subgroup $N$ of $G$ is a simply connected nilpotent Lie group.

We assume now, and throughout the rest of this paper, that $G$ is acceptable (see [4g, §18] for the definition). This assumption enables us to define the following important $C^{\infty}$ functions on $A$ : let $h \in A, h=h_{-} h_{+}$the unique decomposition such that $h_{-} \in A_{-}, h_{+} \in A_{+}$; set

$$
\begin{aligned}
\Delta(h) & =\xi_{\rho}(h) \prod_{\alpha \in P}\left(1-\xi_{\alpha}\left(h^{-1}\right)\right), \\
\Delta_{-}(h) & =\xi_{\rho}\left(h_{-}\right) \prod_{\alpha \in P_{-}}\left(1-\xi_{\alpha}\left(h^{-1}\right)\right), \\
\Delta_{+}(h) & =\Delta(h) / \Delta_{-}(h)=\xi_{\rho}\left(h_{+}\right) \prod_{\alpha \in P_{+}}\left(1-\xi_{\alpha}\left(h^{-1}\right)\right) .
\end{aligned}
$$

We are of course using the notation of $[4 \mathrm{~g}, \S 18]$. Note that

$$
\Delta_{-}(h)=\Delta_{-}\left(h_{-}\right)
$$

(see [4g, Lemma 30] and the definition of $P_{-}$). Also, the roots in $P_{+}$take purely imaginary values on $\mathfrak{h}_{-}$. Therefore it is easy to verify that these functions agree (in absolute value) with the corresponding $\Delta$ functions of [4d].

LEMMA 3.1 (ThE REFEREE). There is a finite subgroup $\Gamma \subseteq A_{-}$such that

(i) $M=M^{\circ} \Gamma$ and $\gamma m^{\circ}=m^{\circ} \gamma$, for all $\gamma \in \Gamma, m^{\circ} \in M^{\circ}$;

(ii) $A=A_{-}^{\circ} \Gamma$;

(iii) for every class function $f$ on $M$

$$
\int_{M} f(m) d m=c \int_{A_{-}} f\left(h_{-}\right)\left|\Delta_{-}\left(h_{-}\right)\right|^{2} d h_{-},
$$

$c$ an absolute constant.

Proof. Let $\Gamma=\operatorname{Ad}_{G}^{-1}\left(\operatorname{Ad}_{G}(K) \cap \exp \sqrt{ }(-1) \mathfrak{h}_{+}\right) . \Gamma$ is easily seen to be a finite subgroup of $A \cap K=A_{-}$whose elements commute with those of $M^{\circ}$. Equation 
(i) is proven by Satake in [6, p. 93]. As for (ii), the fact that $A_{-} \supseteq A_{-}^{\circ} \Gamma$ is obvious. Conversely, let $h \in A_{-}$. Then $h=m \gamma, m \in M^{\circ}, \gamma \in \Gamma$. Hence $m=h \gamma^{-1} \in A_{-} \cap M^{\circ}$ $=A_{-}^{\circ}$, i.e. $h=m \gamma \in A_{-}^{\circ} \Gamma$. More precisely let $n=\#\left(M / M^{\circ}\right)$. We can find $\gamma_{1}, \ldots, \gamma_{n} \in \Gamma$ such that $M=\bigcup_{i=1}^{n} M^{\circ} \gamma_{i}$ (this is the decomposition of $M$ into its connected (open) components). Then we have actually shown that $A_{-}=\bigcup_{i=1}^{n} A_{-}^{\circ} \gamma_{i}$.

Now let $f \in C(M)$. Then

$$
\begin{aligned}
\int_{M} f(m) d m & =\sum_{i=1}^{n} \frac{1}{n} \int_{M^{\circ}} f\left(m^{\circ} \gamma_{i}\right) d m^{\circ} \\
& =c^{\circ} \sum_{i=1}^{n} \int_{A_{-}^{\circ}}\left|\Delta_{-}\left(h_{-}^{\circ}\right)\right|^{2} d h_{-}^{\circ} \int_{M^{\circ}} f\left(m^{\circ} h_{-}^{\circ}\left(m^{\circ}\right)^{-1} \gamma_{i}\right) d m^{\circ} \\
& =c^{\circ} \sum_{i=1}^{n} \int_{A_{-}^{\circ}}\left|\Delta_{-}\left(h_{-}^{\circ}\right)\right|^{2} d h_{-}^{\circ} \int_{M^{\circ}} f\left(m^{\circ} h_{-}^{\circ} \gamma_{i}\left(m^{\circ}\right)^{-1}\right) d m^{\circ} .
\end{aligned}
$$

If $f$ is also a class function (in particular if $f$ is a character), then

$$
\begin{aligned}
\int_{M} f(m) d m & =c \sum_{i=1}^{n} \int_{A_{-}^{\circ}}\left|\Delta_{-}\left(h_{-}^{\circ}\right)\right|^{2} f\left(h_{-}^{\circ} \gamma_{i}\right) d h_{-}^{\circ} \\
& =c \int_{A_{-}} f\left(h_{-}\right)\left|\Delta_{-}\left(h_{-}\right)\right|^{2} d h_{-} .
\end{aligned}
$$

In this calculation the constant factors depend only on normalizations in Haar measure. We also used the fact that

$$
\left|\Delta_{-}\left(h_{-}^{\circ} \gamma_{i}\right)\right|=\left|\Delta_{-}\left(h_{-}^{\circ}\right)\right| .
$$

This is true because $\gamma \rightarrow\left|\xi_{\rho}(\gamma)\right|$ is a homomorphism of the finite group $\Gamma$ into $\boldsymbol{R}^{+}$, and $\xi_{\alpha}(\gamma)=1$ whenever $\alpha \in P_{-}$. This completes the proof of (iii) and the lemma (compare also [4g, Lemmas 50 and 51$]$ ).

Let $G_{1}=$ the regular elements in $G$ (see [4d, p. 504] for the definition) and set $A_{1}=A \cap G_{1}, G_{A}=A^{G} \cap G_{1} . G_{A}$ consists of the regular elements that conjugate into $A$, or more precisely those that conjugate into $A_{1}$. Moreover $A_{1}=\{h \in A: \Delta(h) \neq 0\}$ is a dense open submanifold of $A$. If we extend $\Delta^{-1}$ from $A_{1}$ to $G_{A}$ by conjugation and set it equal to zero off $G_{A}$, the resulting function is known to be locally integrable on $G$ (see $[4 \mathrm{~g}, \S 28]$ ).

Finally let $A_{0}=$ the center of $A$. Consider the finite group $W_{A}=A^{\prime} / A_{0}=$ $A_{-}^{\prime} /\left(A_{0} \cap K\right)$, where $A_{-}^{\prime}=N(\mathfrak{h}) \cap K$ and $A^{\prime}=N(\mathfrak{h})=A_{-}^{\prime} A_{+}$. Consider also the continuous mapping $\phi: G / A_{0} \times A \rightarrow G, \phi(\bar{x}, h)=h^{x}=x h x^{-1}, \bar{x}=x A_{0}, x \in G$. Then the restricted map

$$
\phi: G / A_{0} \times A_{1} \rightarrow G_{A}
$$

is regular and onto-so $G_{A}$ is open. The complete inverse image of a point $h^{x}$ in $G_{A}$ is precisely the \#( $\left.W_{A}\right)$ distinct points $\left\{\left(\bar{x} s^{-1}, h^{s}\right): s \in W_{A}\right\}$, where $h^{s}=y h y^{-1}$ and $\bar{x} s^{-1}=\left(x y^{-1}\right)^{-}, y$ a representative of $s$ in $A^{\prime}$. That is $W_{A}$ acts on $G / A_{0} \times A_{1}$ without fixed points. Hence $G_{A}$ may be identified (via $\phi$ ) with the quotient space 
$W_{A} \mid\left(G / A_{0} \times A_{1}\right)$. As a consequence, we see that the inverse image under $\phi$ of a compact set in $G_{A}$ is compact in $G / A_{0} \times A_{1}$. This will be useful in $\S 5$. We also note that $W_{A}$ acts as a group of linear transformations on $\mathfrak{h}$ by $s H=\operatorname{Ad} y(H), H \in \mathfrak{h}$, $s \in W_{A}, y$ a representative of $s$ in $A^{\prime}$. Both $\mathfrak{h}_{-}$and $\mathfrak{h}_{+}$are invariant under this action.

The reader may find the results of this section which are stated without proof in [4a], [4d], and [4g].

4. The principal series. We identify the set $\hat{A}_{+}$with the set of all real linear functionals $\nu: \mathfrak{h}_{+} \rightarrow \boldsymbol{R}$ via $\nu(h) \equiv \exp i \nu(H), h=\exp H, H \in \mathfrak{h}_{+}, h \in A_{+} . W_{A}$ acts on $\hat{A}_{+}$by $s \nu(H)=\nu\left(s^{-1} H\right), s \in W_{A}, H \in \mathfrak{h}_{+}$. The group $W_{A}$ also acts on the set $\hat{M}$ as follows: for $\delta \in \hat{M}$, let $s \delta(m)=\delta\left(u^{-1} m u\right), m \in M, s \in W_{A}$ and $u$ a representative of $s$ in $A_{-}^{\prime}$.

Corresponding to each $\nu \in \hat{A}_{+}, \delta \in \hat{M}$, there exists a unitary representation $T_{v, \delta}$ of $G$ obtained by inducing the finite-dimensional representation $m h_{+} n \rightarrow$ $\nu\left(h_{+}\right) \delta(m)$ of $M A N$ to the group $G$. These constitute the principal series (see e.g. [1]). The representations $T_{v, \delta}$ have the following property: If $f \in C_{0}^{\infty}(G)$, then $\int_{G} f(g) T_{v, \delta}(g) d g$ is a trace class operator and $f \rightarrow \tau_{v, \delta}(f)=\operatorname{Tr}\left(\int_{G} f(g) T_{v, \delta}(g) d g\right)$ is a distribution. Indeed there exist locally integrable functions $\theta_{v, \delta}$ on $G$ such that

$$
\tau_{v, \delta}(f)=\int_{G} f(g) \theta_{v, \delta}(g) d g, \quad f \in C_{0}^{\infty}(G) .
$$

The functions $\theta_{v, \delta}$ have been computed by Harish-Chandra in [4d, Theorem 2]:

$$
\begin{aligned}
& \theta_{v, \delta}(x)=0 \quad \text { if } x \notin G_{A}, \quad \theta_{v, \delta}\left(y x y^{-1}\right)=\theta_{v, \delta}(x) \quad \text { if } x \in G_{A}, y \in G, \\
& \theta_{v, \delta}(h)=c_{0}\left|\Delta_{+}(h)\right|^{-1} \sum_{s \in W_{A}} \exp \left\{i s v\left(\log h_{+}\right)\right\} \xi_{s \delta}\left(h_{-}\right), \quad h \in A_{1} .
\end{aligned}
$$

Here $c_{0}$ is an absolute constant, $h=h_{-} h_{+}, h_{-} \in A_{-}, h_{+} \in A_{+}$, and $\xi_{\delta}$ is the character of the representation $\delta$. It is obvious that $\theta_{v, \delta}$ is analytic on $G_{1}$.

RemarK. Harish-Chandra's original computation of the characters $\theta_{v, \delta}$ is carried out only under the assumption $M=M^{\circ} Z, Z=$ Cent $(G)$. This is the additional technical assumption (referred to in the introduction) that we made in the earlier version of this paper. The sole reason for this is the need to use Weyl's integration formula [4d, p. 497]. But Lemma 3.1 frees us from this restriction now. Using it in the appropriate place in [4d, Lemma 6], we see that the character formula (4.2) is valid for any connected semisimple Lie group which has finite center and is acceptable. We will have occasion to use Lemma 3.1 again in the proofs of Lemmas 5.2, 5.3, 5.4, and 8.1.

We recall now some of Bruhat's fundamental results. Let $M^{\prime}=N\left(A_{+}\right) \cap K$ $=N\left(\mathfrak{h}_{+}\right) \cap K$. Then $W_{B}=M^{\prime} \mid M$ is a finite group which acts on $\hat{A}_{+}$and $\hat{M}$ :

$$
\begin{aligned}
s v(h) & =v\left(u^{-1} h u\right), & & h \in A_{+}, \\
s \delta(m) & =\delta\left(u^{-1} m u\right), & & m \in M,
\end{aligned}
$$

$s \in W_{B}$ and $u$ any representative of $s$ in $M^{\prime}$. 
LeMma $4.1[1, \S 7.4]$. (1) $T_{v, \delta}$ is unitarily equivalent to $T_{v^{\prime}, \delta^{\prime}}$ if and only if there exists $s \in W_{B}$ such that $\nu=s \nu^{\prime}, \delta \cong s \delta^{\prime}$.

(2) If $I(T, S)$ denotes the dimension of the intertwining space for two unitary representations $T$ and $S$, then $I\left(T_{v, \delta}, T_{v^{\prime}, \delta^{\prime}}\right) \leqq \# s \in W_{B}$ such that $\nu=s \nu^{\prime}$ and $\delta \cong s \delta^{\prime}$.

Before stating the natural consequences of this result, we first prove

LEMMA 4.2. There exists an absolute constant $c_{1}$ such that

$$
\sum_{s \in W_{A}} \exp \left\{i s v\left(\log h_{+}\right)\right\} \xi_{s \delta}\left(h_{-}\right)=c_{1} \sum_{s \in W_{B}} \exp \left\{i s v\left(\log h_{+}\right)\right\} \xi_{s \delta}\left(h_{-}\right),
$$

for all $h=h_{-} h_{+} \in A$.

Proof. We have

$$
W_{B}=\frac{N\left(\mathfrak{h}_{+}\right) \cap K}{Z\left(\mathfrak{h}_{+}\right) \cap K}=\frac{\left[N\left(\mathfrak{h}_{+}\right) \cap K\right] \cdot A_{+}}{\left[Z\left(\mathfrak{h}_{+}\right) \cap K\right] \cdot A_{+}}=\frac{N\left(\mathfrak{h}_{+}\right)}{Z\left(\mathfrak{h}_{+}\right)}
$$

But suppose we can prove

$$
N\left(\mathfrak{h}_{+}\right)=Z\left(\mathfrak{h}_{+}\right) N(\mathfrak{h})
$$

Then

$$
W_{B}=\frac{N(\mathfrak{h})}{Z(\mathfrak{h}+) \cap N(\mathfrak{h})}=\frac{N(\mathfrak{h}) / Z(\mathfrak{h})_{0}}{[Z(\mathfrak{h}+) \cap N(\mathfrak{h})] / Z(\mathfrak{h})_{0}}=\frac{W_{A}}{W_{0}}
$$

where $W_{0}=\left[Z\left(\mathfrak{h}_{+}\right) \cap N(\mathfrak{h})\right] / Z(\mathfrak{h})_{0}=\left[Z\left(A_{+}\right) \cap A^{\prime}\right] / A_{0}$.

Obviously $W_{0}$ acts trivially on $\mathfrak{h}_{+}$and $\hat{A}_{+}$. Although $W_{0}$ acts nontrivially on $M$-in fact, by inner automorphism-it therefore acts trivially on $\hat{M}$. The lemma follows then with $c_{1}=\#\left(W_{0}\right)$.

Proof of (4.3). Clearly $Z\left(\mathfrak{h}_{+}\right) \subseteq N\left(\mathfrak{h}_{+}\right)$. Let $x \in N(\mathfrak{h}) \cap K$. Then Ad $x \mathfrak{h}_{+} \subseteq \mathfrak{p}$ and Ad $x \mathfrak{h}_{+} \subseteq \operatorname{Ad} x \mathfrak{h} \subseteq \mathfrak{h}$. Hence $\mathfrak{h}_{+}^{x} \subseteq \mathfrak{h} \cap \mathfrak{p}=\mathfrak{h}_{+}$. That is, $x \in N\left(\mathfrak{h}_{+}\right)$. Since $N(\mathfrak{h})$ $=[N(\mathfrak{h}) \cap K] \cdot A_{+}$and $A_{+} \subseteq N\left(\mathfrak{h}_{+}\right)$, it follows that $N\left(\mathfrak{h}_{+}\right) \supseteq Z\left(\mathfrak{h}_{+}\right) N(\mathfrak{h})$.

Conversely, let $x \in N\left(\mathfrak{h}_{+}\right) \cap K$. Since $\mathfrak{m}$ is the centralizer of $\mathfrak{h}_{+}$in $\mathfrak{t}$, it follows easily that $x \in N(\mathfrak{m})$. Hence $\mathfrak{h}_{-}^{x}$ is a Cartan subalgebra of $\mathfrak{m}$. We can therefore choose $m \in M^{\circ}$ so that $\mathfrak{h}_{-}=\mathfrak{h}_{-}^{m x}$. That is, $m x \in N\left(\mathfrak{h}_{-}\right)$. But then we have $m x \in N(\mathfrak{h})$ or $x \in Z\left(\mathfrak{h}_{+}\right) N(\mathfrak{h})$. We conclude by observing $N\left(\mathfrak{h}_{+}\right)=\left[N\left(\mathfrak{h}_{+}\right) \cap K\right] \cdot A_{+}$and $A_{+} \subseteq Z\left(\mathfrak{h}_{+}\right) N(\mathfrak{h})$.

Note. We actually proved $N\left(\mathfrak{h}_{+}\right)=Z\left(\mathfrak{h}_{+}\right)^{\circ} N(\mathfrak{h})$.

The equation of the character on $A_{1}$ may now be written

$$
\theta_{v, \delta}(h)=c\left|\Delta_{+}(h)\right|^{-1} \sum_{s \in W_{B}} \exp \left\{i s v\left(\log h_{+}\right)\right\} \xi_{s \delta}\left(h_{-}\right), \quad h \in A_{1} .
$$

Henceforth, we shall consider only $W_{B}$ and so for convenience we drop the subscript $B$.

Definition. $(\nu, \delta) \in \hat{A}_{+} \times \hat{M}$ is said to be in general position if for every $s \in W$, $s \neq 1$, we have $s(\nu, \delta) \not(\nu, \delta)$, i.e. either $s \nu \neq \nu$ or $s \delta \not \delta$ or both. 
Lemma 4.3. If $(\nu, \delta)$ is in general position, then $T_{v, \delta}$ is irreducible. Suppose $(\nu, \delta)$ is not in general position. Then either (i) $T_{v, \delta}$ is irreducible, or (ii) there exist at least 2 and at most $\#(W)$ irreducible unitary representations $T^{j}$ of $G$ (not necessarily distinct a priori) such that $T_{v, \delta}=\oplus T^{j}$; moreover, no $T^{j}$ is unitarily equivalent to any member of the irreducible principal series.

Proof. This lemma follows immediately from Lemma 4.1. With the exception of the last statement, Bruhat made this observation in [1, p. 193].

But suppose $T^{j} \cong T_{v^{\prime}, \delta^{\prime}}$ for some $j$ and some $\left(v^{\prime}, \delta^{\prime}\right)$ such that $T_{v^{\prime}, \delta^{\prime}}$ is irreducible. If there exists $s \in W$ such that $s(\nu, \delta) \cong\left(\nu^{\prime}, \delta^{\prime}\right)$, then $T_{v, \delta} \cong T_{v^{\prime}, \delta^{\prime}} \Rightarrow T_{v, \delta}$ is irreducible, contrary to hypothesis. On the other hand, if no $s \in W$ exists, then $I\left(T_{v, \delta}, T_{v^{\prime}, \delta^{\prime}}\right)=0$. This says $T_{v, \delta}$ and $T_{v^{\prime}, \delta^{\prime}}$ are disjoint and contradicts the occurrence of $T_{v^{\prime}, \delta^{\prime}}$ as a direct summand of $T_{v, \delta}$.

It follows from [3, Theorem 1.5] that the hull-kernel topology on $\hat{A}_{+}$agrees with the usual topology it inherits as the character group of a locally compact abelian group. Also, since $M$ is compact, the hull-kernel topology of $\hat{M}$ is the discrete topology (because of $[2,18.4 .3]$ ). Hence we impose on $\hat{A}_{+} \times \hat{M}$ the product topology of the Euclidean space $\hat{A}_{+}$with the discrete topology of $\hat{M}$. Consider the orbit space $\mathscr{Q}$ of $\hat{A}_{+} \times \hat{M}$ under the action of the finite group $W$. It is easy to check that the quotient topology on $\mathscr{Q}=W \backslash\left(\hat{A}_{+} \times \hat{M}\right)$ is locally compact, Hausdorff, and second countable. In addition, since the characters satisfy $\theta_{v, \delta}=\theta_{s v, s \delta}, s \in W$, we may associate a character $\theta_{q}$ to each $q \in \mathscr{Q}, q=W \cdot(\nu, \delta)$.

We single out the subset of irreducible members of 2 . Let

$$
\mathscr{P}=\left\{q=W \cdot(\nu, \delta) \in \mathscr{Q}: T_{\nu, \delta} \text { is irreducible }\right\} .
$$

In general $\mathscr{P} \varsubsetneqq \mathscr{Q}$; however, it is clear from Lemma 4.3 that $\mathscr{P}$ is dense in $\mathscr{Q}$ in the topology we just defined. Now it follows from the previous arguments that $\mathscr{P}$ is embedded in $\hat{G}$. Hence $\mathscr{P}$ has two topologies: the natural topology as a subspace of $\mathscr{Q}$ and the hull-kernel topology induced by $\hat{G}$. One of our main results will be that they are the same.

5. Basic lemmas. We prove here two results (Lemmas 5.1 and 5.2) which will be fundamental to our work in the last three sections.

LEMMA 5.1. Let $\left\{q^{i}\right\}$ be a net in $\mathscr{Q}$ and $q \in \mathscr{Q}$ such that $q^{i} \rightarrow q$ in the natural topology. Then for each $f \in C_{0}(G)$,

$$
\int f(g) \theta_{q}(g) d g \rightarrow \int f(g) \theta_{q}(g) d g .
$$

Proof. Since $\mathscr{Q}$ is second countable, we may assume the net is a sequence. If $q=W \cdot(\nu, \delta)$, it is clear from the topology that $q^{i}=W \cdot\left(\nu^{i}, \delta\right)$ for $i$ sufficiently large. But for those $i$,

$$
\left|\theta_{q^{i}}\right| \leqq c \#(W)(\operatorname{deg} \delta) \Delta_{+}^{-1},
$$

a locally integrable function on $G$. Moreover, it follows from (4.2) and (4.4) that 
for any fixed $g \in G, \theta_{q^{i}}(g) \rightarrow \theta_{q}(g)$. The lemma follows by an appeal to the Lebesgue dominated convergence theorem.

LEMma 5.2. Let $\left\{q^{i}\right\}$ be a net in $\mathscr{Q}$ converging in the natural topology to the point at infinity. Then for each $f \in C_{0}^{\infty}(G)$,

$$
\int f(g) \theta_{q^{i}}(g) d g \rightarrow 0
$$

Proof. We may assume again that the net is a sequence, say $q^{n}=W \cdot\left(\nu^{n}, \delta^{n}\right)$. First suppose that $f \in C_{0}^{\infty}\left(G_{1}\right)$, i.e. the support of $f$ is contained in $G_{1}$. It follows from $[4 \mathrm{~g}$, Lemma 41] that

$$
\int_{G} f(g) \theta_{q^{n}}(g) d g=c_{A} \int_{A}|\Delta(h)|^{2} d h \int_{G^{*}} f\left(h^{\bar{g}}\right) \theta_{q^{n}}\left(h^{\bar{g}}\right) d \bar{g},
$$

where $G^{*}=G / A_{0}, d \bar{g}$ is the $G$-invariant measure on $G^{*}$ and $d h=$ the bi-invariant measure on $A$ (it exists since $A / A_{0}$ is finite). By (4.2), (4.4) and (3.1), we get

$$
\begin{aligned}
\int f(g) & \theta_{q^{n}}(g) d g \\
= & c c_{A} \int_{A}|\Delta(h)|^{2}\left|\Delta_{+}(h)\right|^{-1} \sum_{s \in W} \exp \left\{i s \nu^{n}\left(\log h_{+}\right)\right\} \xi_{s \delta^{n}}\left(h_{-}\right) d h \int_{G^{*}} f\left(h^{\bar{\delta}}\right) d \bar{g} \\
& =\sum_{s \in W} \int_{A}\left|\Delta_{-}\left(h_{-}\right)\right|^{2} \exp \left\{i s \nu^{n}\left(\log h_{+}\right)\right\} \xi_{s \delta^{n}}\left(h_{-}\right) F(h) d h,
\end{aligned}
$$

where $F(h)=c c_{A}\left|\Delta_{+}(h)\right| \int_{G^{*}} f\left(h^{\bar{g}}\right) d \bar{g}$.

For general $f \in C_{0}^{\infty}(G), F$ is a function of compact support in $A$ and $C^{\infty}$ on $A_{1}$. But it may have "jumps" or "corners" across the singular set where $\Delta$ vanishes (see $[4 \mathrm{e}, \S \S 1,4]$ ). By restricting the support of $f$ to $G_{1}$, we insure (by the remarks at the end of $\S 3$ ) that Supp $F \subseteq A_{1}$. Hence $F \in C_{0}^{\infty}\left(A_{1}\right)$, and so canonically $F \in C_{0}^{\infty}(A)$.

Fix $s \in W$ and denote $s \nu^{n}=\nu, s \delta^{n}=\delta$. By (5.1) it is enough to show

$$
\int_{A} \exp \left\{i v\left(\log h_{+}\right)\right\} \xi_{\delta}\left(h_{-}\right)\left|\Delta_{-}\left(h_{-}\right)\right|^{2} F(h) d h \rightarrow 0
$$

as $(\nu, \delta) \rightarrow \infty$ in $\hat{A}_{+} \times \hat{M}$. We extend the function $F$ to all of $M A_{+}$by conjugation (every element of $M=M^{\circ} \Gamma$ is conjugate to an element in $A_{-}=A_{-}^{\circ} \Gamma$ ). Since $P_{-}$may be identified with the positive roots of $\left(\mathfrak{m}, \mathfrak{h}_{-}\right)$, we see again that the support of the resulting function is bounded away from the singular set; and so $F \in C_{0}^{\infty}\left(M A_{+}\right)$.

We now use Lemma 3.1:

$$
\begin{aligned}
\int_{A} \exp \left\{i v\left(\log h_{+}\right)\right\} \xi_{\delta}\left(h_{-}\right)\left|\Delta_{-}\left(h_{-}\right)\right|^{2} F(h) d h \\
=\int_{A_{+}} \exp \left\{i \nu\left(\log h_{+}\right)\right\} d h_{+} \int_{A_{-}}\left|\Delta_{-}\left(h_{-}\right)\right|^{2} \xi_{\delta}\left(h_{-}\right) F\left(h_{-} h_{+}\right) d h_{-} \\
=\int_{A_{+}} \exp \left\{i \nu\left(\log h_{+}\right)\right\} d h_{+} \int_{M} \xi_{\delta}(m) F\left(m h_{+}\right) d m ;
\end{aligned}
$$

and so we need to prove 
Lemma 5.3. Let $F \in C_{0}^{\infty}\left(M A_{+}\right)$. Then as $(\nu, \delta) \rightarrow \infty$ in the natural topology of $\hat{A}+\times \hat{M}$, we have

$$
\int_{M A_{+}} \exp \left\{i v\left(\log h_{+}\right)\right\} \xi_{\delta}(m) F\left(m h_{+}\right) d m d h_{+} \rightarrow 0 .
$$

Proof. This lemma is likely known, although perhaps not in this context. The proof is from classical representation theory of abelian and compact Lie groups.

First assume $M$ is connected. Let $\Omega=$ the Casimir operator on $M A_{+}$. The functions $e^{i v(\cdot)} \xi_{\delta}(\cdot)$, being the characters of the irreducible unitary representations of $M A_{+}$, are eigenfunctions of $\Omega$. The computation of the eigenvalue is fairly standard. Let $\Omega_{M}=$ the Casimir operator on $M$ and $\Omega_{A_{+}}=$the Casimir operator (i.e. Laplacian) on $A_{+}$. Then $\Omega=\Omega_{M}+\Omega_{A_{+}}$. If $\|\cdot\|_{+}$denotes a suitable Euclidean norm on $\mathfrak{h}_{+}^{*}$ (e.g. the one determined by the Killing form), then $\Omega_{A_{+}} e^{i v(\cdot)}=-\|\nu\|_{+}^{2} e^{i v(\cdot)}$. Since $M$ is connected, we can identify $\hat{M}$ with the finite-dimensional irreducible representations of $\mathfrak{m}_{c} . \delta \in \hat{M}$ is then uniquely determined by a highest weight $\Lambda$ and $\Omega_{M} \xi_{\delta}=\Lambda\left(\Omega_{M}\right) \xi_{\delta}$. If we fix a system of positive roots on $\left(\mathfrak{m}, \mathfrak{h}_{-}\right)$, we may realize

$$
\Omega_{M}=-H_{1}^{2}-\cdots-H_{r}^{2}+\sum_{\beta>0} X_{\beta} X_{-\beta}+X_{-\beta} X_{\beta},
$$

where the $X_{\beta}$ are root vectors and the $H_{i}$ form a basis of $\mathfrak{h}_{-}$chosen so that with respect to the Killing form $\left(H_{i}, H_{j}\right)=-\delta_{i j}$. Letting $H_{\beta}=\left[X_{\beta}, X_{-\beta}\right] \in\left(\mathfrak{h}_{-}\right)_{c}$ and $2 \rho=\sum_{\beta>0} \beta$, we see that

$$
\Omega_{M}=-H_{1}^{2}-\cdots-H_{r}^{2}+\sum_{\beta>0} 2 X_{-\beta} X_{\beta}+H_{\beta} .
$$

Then

$$
\begin{aligned}
\Lambda\left(\Omega_{M}\right) & =-\sum_{i=1}^{r} \Lambda\left(H_{i}\right)^{2}+\sum_{\beta>0} \Lambda\left(H_{\beta}\right) \\
& =(\Lambda, \Lambda)+2(\Lambda, \rho) \\
& =-\|\Lambda+\rho\|_{-}^{2}+\|\rho\|_{-}^{2},
\end{aligned}
$$

where $\|\cdot\|_{-}$is the positive definite form on $\mathfrak{h}_{-}^{*}$ determined by the Killing form negated. We have shown that

$$
\Omega\left(e^{i v(\cdot)} \xi_{\delta}(\cdot)\right)=\left(-\|\nu\|_{+}^{2}-\|\Lambda+\rho\|_{-}^{2}+\|\rho\|_{-}^{2}\right) e^{i v(\cdot)} \xi_{\delta}(\cdot) .
$$

Let $d \in Z^{+}$and let $\omega=1+\|\rho\|_{-}^{2}-\Omega$. Using integration by parts, we obtain

$$
\begin{aligned}
\operatorname{deg} \delta\left\|\omega^{d} F\right\|_{1} & \geqq\left|\int e^{i v} \xi_{\delta} \omega^{d} F\right| \\
& =\left|\int \omega^{d}\left(e^{i v} \xi_{\delta}\right) F\right| \\
& =\left(1+\|\nu\|_{+}^{2}+\|\Lambda+\rho\|_{-}^{2}\right)^{d}\left|\int e^{i v} \xi_{\delta} F\right|
\end{aligned}
$$


But Weyl's classical formula gives $\operatorname{deg} \delta$ as a polynomial $p(\Lambda)$ in the highest weight $\Lambda$; indeed

Hence

$$
p(\Lambda)=\prod_{\beta>0}(\Lambda+\rho, \beta) / \prod_{\beta>0}(\rho, \beta) .
$$

$$
\left|\int e^{i v} \xi_{\delta} F\right| \leqq p(\Lambda)\left(1+\|\nu\|_{+}^{2}+\|\Lambda+\rho\|_{-}^{2}\right)^{-d}\left\|\omega^{d} F\right\|_{1} .
$$

It is clear that $\delta \rightarrow \infty \Rightarrow \Lambda \rightarrow \infty$, and so for $d$ sufficiently large the right side of (5.3) must tend to zero as $(\nu, \delta) \rightarrow \infty$.

Now in the general case, Lemma 3.1 insures that $M=M^{\circ} \Gamma$. Let $\delta \in \hat{M}$ and consider $\left.\delta\right|_{M^{\circ}}$. In general, it is not irreducible; but since $\Gamma$ and $M^{\circ}$ commute it is easy to see that $\left.\delta\right|_{M^{\circ}}$ must be a multiple of a single irreducible representation $\delta^{\circ} \in\left(M^{\circ}\right)^{\wedge},\left.\delta\right|_{M^{\circ}}=n_{\delta \mid \delta^{\circ}} \delta^{\circ}$. Of course $n_{\delta \mid \delta^{\circ}} \leqq \#(\Gamma)$; so $\operatorname{deg} \delta \leqq \#(\Gamma) p(\Lambda), \Lambda=$ the highest weight of $\delta^{\circ}$. The Casimir operator defined by (5.2) acts canonically on $M$. Moreover the character $\xi_{\delta}$ will again be an eigenfunction with the same eigenvalue. (This can be seen by examining the associated representation of the universal enveloping algebra of $\mathfrak{m}$.) It is a simple matter now to complete the argument.

To finish the proof of Lemma 5.2 we need to handle an arbitrary $f \in C_{0}^{\infty}(G)$. For that we first prove the following.

Lemma 5.4. There exists an absolute constant $C$ such that for every $(\nu, \delta) \in \hat{A}_{+} \times \hat{M}$,

$$
\sup _{h \in A}\left(\left|\Delta(h) \theta_{v, \delta}(h)\right|\right) \leqq C .
$$

Proof. Assume $M$ is connected. By Weyl's character formula, if $\Lambda$ is the highest weight corresponding to $\delta \in \hat{M}$, then

$$
\xi_{\delta}\left(h_{-}\right)=\frac{1}{\Delta_{1}\left(h_{-}\right)} \sum_{s \in W_{1}} \varepsilon(s) \exp (s(\Lambda+\rho)(H)), \quad h_{-} \in A_{-}^{\circ} .
$$

Here the epsilons are \pm 1 (the actual definition is not important for us), $\exp H=h_{-}$, $\rho$ is as in Lemma 5.3, $W_{1}=W\left(\mathfrak{m}, \mathfrak{h}_{-}\right), \Delta_{1}\left(h_{-}\right)=\prod_{\alpha \in P_{-}}\left(e^{\alpha(H) / 2}-e^{-\alpha(H) / 2}\right)$ and $\left|\Delta_{1}\right|=\left|\Delta_{-}\right|$. The lemma is now obvious from (4.4), (5.5), and the fact that $\Lambda+\rho$ is purely imaginary on $\mathfrak{h}_{-}$.

One may treat the case $M=M^{\circ} \Gamma$ by considering Kostant's generalization of Weyl's character formula to disconnected groups. However (as pointed out to me by my colleague A. Kleppner), there is a much more elementary method. Since $M=M^{\circ} \Gamma$ with $M^{\circ}$ and $\Gamma$ commuting, $M$ is isomorphic to $\left(M^{\circ} \times \Gamma\right) / \Gamma_{1}, \Gamma_{1}=$ $\left\{\left(\gamma, \gamma^{-1}\right): \gamma \in M_{0} \cap \Gamma\right\}$. Then $\hat{M}=\left[\left(M^{\circ} \times \Gamma\right) / \Gamma_{1}\right]^{\wedge}=\left\{\delta \in\left(M^{\circ} \times \Gamma\right)^{\wedge}: \delta \mid \Gamma_{1}=I\right\}$. Since $\left(M^{\circ} \times \Gamma\right)^{\wedge}=\left(M^{\circ}\right)^{\wedge} \times \hat{\Gamma}$, we can say the following: If $\delta \in \hat{M}$, there are (uniquely determined) irreducible representations $\delta^{\circ} \in\left(M^{\circ}\right)^{\wedge}, \varepsilon \in \hat{\Gamma}$, such that $\left.\delta^{\circ}\right|_{M^{\circ} \cap \Gamma},\left.\varepsilon\right|_{M^{\circ} \cap \Gamma}$ act by the same scalar operator and $\delta\left(m^{\circ} \gamma\right)=\delta^{\circ}\left(m^{\circ}\right) \otimes \varepsilon(\gamma)$, $m^{\circ} \in M^{\circ}, \gamma \in \Gamma$. Therefore

$$
\xi_{\delta}\left(m^{\circ} \gamma\right)=\xi_{\delta^{\circ}}\left(m^{\circ}\right) \xi_{\varepsilon}(\gamma), \quad m^{\circ} \in M^{\circ}, \gamma \in \Gamma .
$$


Of course $\operatorname{deg} \varepsilon \leqq \#(\Gamma)$ and so $\left|\xi_{\delta}\left(m^{\circ} \gamma\right)\right| \leqq \#(\Gamma)\left|\xi_{\delta^{\circ}}\left(m^{\circ}\right)\right|$. Applying (5.5) to $\xi_{\delta^{\circ}}$ and using (3.2), we see again that (5.4) must hold. (Note. It also follows from (5.6) that $\xi_{\delta}$ is an eigenfunction of the Casimir operator with the same eigenvalue as $\xi_{\delta^{\circ}}$.)

Completion of the proof of Lemma 5.2. Note (it follows by conjugation and the fact that $\theta_{v, \delta}=0$ off $G_{A}$ ) that (5.4) actually holds on all of $G$.

Let $f \in C_{0}^{\infty}(G), S=\operatorname{Supp} f$ and $\varepsilon>0$. We can choose a compact set $S_{1} \subseteq G_{1}$ such that $\int_{S-S_{1}}|\Delta|^{-1}<\varepsilon$. Moreover we can choose $f_{1} \in C_{0}^{\infty}\left(G_{1}\right)$ such that $\left|f_{1}\right| \leqq|f|$ and $f=f_{1}$ on $S_{1}$. But then for $i$ sufficiently large $\left|\int f_{1} \theta_{q^{i}}\right|<\varepsilon$. Hence

$$
\begin{aligned}
\left|\int f \theta_{q^{i}}\right| & \leqq\left|\int\left(f-f_{1}\right) \theta_{q^{i}}\right|+\left|\int f_{1} \theta_{q^{i}}\right| \\
& \leqq C \int_{S-S_{1}}\left|f-f_{1}\right||\Delta|^{-1}+\varepsilon \\
& \leqq \varepsilon\left(1+2 C \sup _{g \in G}|f(g)|\right) .
\end{aligned}
$$

RemarK. In the event that $G$ is complex, $M=A_{-}$is a torus and $M A_{+}=A$ is abelian. Lemma 5.3 in this case is a consequence of the abstract Riemann-Lebesgue Lemma on $A$ (see the proof of [3, Lemma 3.9] in this regard). In the general case, by $[2,18.2 .4]$, it is true that for any $F \in L_{1}\left(M A_{+}\right)$,

$$
\left\|\int_{M A_{+}} \exp \left(i \nu\left(\log h_{+}\right)\right) \delta(m) F\left(m h_{+}\right) d m d h_{+}\right\|_{\infty} \rightarrow 0
$$

as $(\nu, \delta) \rightarrow \infty$ in $\hat{A}_{+} \times \hat{M}$. However, this does not imply Lemma 5.3. The trace of the above operator may be larger in absolute value than the operator norm. Therefore we must employ a proof as above and restrict ourselves to $C_{0}^{\infty}$ functions.

6. The topology of $\mathscr{P}$. Suppose $q \in \mathscr{Q}-\mathscr{P}$. Then $T_{q}$ (i.e. $T_{v, \delta}$ for any $(\nu, \delta) \in q$ ) is reducible and $T_{q}=\oplus T^{j}$, a finite direct sum of irreducible unitary representations. Let $\mathscr{R}=$ the collection of all those irreducible unitary representations of $G$ which arise as direct summands of representations $T_{q}, q \in \mathscr{Q}-\mathscr{P}$. By Lemma 4.3, $\mathscr{P} \cap \mathscr{R}=\varnothing$; and so $\mathscr{P} \cup \mathscr{R}$ is embedded in $\hat{G}$.

REMARK. Of course, we identify unitarily equivalent members of $\mathscr{R}$. However, note that by Lemma 4.1 part (2), if $q_{1}, q_{2} \in \mathscr{Q}-\mathscr{P}$ and $q_{1} \neq q_{2}$, then no direct summand of $T_{q_{1}}$ is unitarily equivalent to any direct summand of $T_{q_{2}}$. We should mention also that the following question is still open: Given $q \in \mathscr{Q}-\mathscr{P}, T_{q}=\bigoplus_{j=1}^{r} T^{j}$, does $1 \leqq i \neq j \leqq r \Rightarrow T^{i} \not T^{j}$ ?

If $T \in \mathscr{R}$, then [4c, §5] shows that $T_{f}=\int f(g) T_{g} d g$ is a trace class operator for every $f \in C_{\mathrm{c}}^{\infty}(G)$. Moreover, there exists a locally integrable function $\theta$ on $G$, analytic on $G_{1}$, such that $\operatorname{Tr}\left(T_{f}\right)=\int f(g) \theta(g) d g, f \in C_{0}^{\infty}(G)$ (see [4g, Theorem 2]). It is trivial to check:

LEMMA 6.1. $T_{q}=\bigoplus T^{j} \Rightarrow \theta_{q}=\sum \theta_{j}$ a.e., where $\theta_{j}$ is the character corresponding to $T^{j}$.

We come now to one of our main results. 
THEOREM 6.2. With each point $q \in \mathscr{Q}$ we associate one or more points of $\mathscr{P} \cup \mathscr{R}$ as follows:

(i) If $q \in \mathscr{P}$, with $q$ is associated just $q$ itself.

(ii) If $q \in \mathscr{Q}-\mathscr{P}$, with $q$ are associated those $T \in \mathscr{R}$ that occur as direct summands of $T_{q}$.

Then for any subset $\mathscr{S} \subseteq \mathscr{P}$, the hull-kernel closure of $\mathscr{S}$ in $\hat{G}$ consists precisely of those $p \in \mathscr{P} \cup \mathscr{R}$ which are associated with the points $q$ in the natural closure of $\mathscr{S}$ in 2.

Proof. We denote by $\&$ the family of all minimal central functions in $L_{2}(K)$, i.e. $\mathfrak{F}=\left\{\operatorname{dim} \delta \cdot \xi_{\delta}: \delta \in R\right\}, \xi_{\delta}=$ the character of the representation $\delta$. Then the subalgebra $\mathfrak{A}$ of Lemma 2.2 is exactly the set of all finite linear combinations of elements in $\& * C^{*}(G) *$ E. This says roughly that $K$ is a "large compact subgroup" of $G$.

In fact, the proof that this $\mathfrak{A}$ satisfies the conditions of Lemma 2.2 reduces easily to showing: There is a complete system $\Omega$ of completely irreducible representations of $G$ and an integer $N \geqq 1$ such that for every $T \in \Omega, d \in R$,

$$
\#(T, \delta) \leqq N \cdot \operatorname{dim} \delta,
$$

where \#(T, $\delta)$ is the number of times $\delta$ is contained in $T$. If $G$ has a faithful matrix representation, then $\Omega$ can be chosen to be the set of all finite-dimensional irreducible representations of $G$. In fact Fell makes this assumption in [3]. In general let $G$ be any connected semisimple Lie group with finite center. If we take $\Omega=$ all quasisimple irreducible representations of $G$, then Harish-Chandra has computed $N$ such that (6.1) holds for all $T \in \Omega, \delta \in R$ (see for example [4c, Theorem 4]). This allows us to drop the matrix group assumption.

Since $C_{0}^{\infty}(G)$ is dense in $C^{*}(G)$, the set $\mathfrak{B}$ of all finite linear combinations of functions in $\mathbb{E} * C_{0}^{\infty}(G) * \mathbb{E}$ is dense in $\mathfrak{A}$. Hence, $\mathfrak{B}$ is a dense selfadjoint subalgebra of $C^{*}(G)$ all of whose elements are boundedly represented in $\hat{G}$. We remark that elements of $\mathfrak{B}$ are also $C_{0}^{\infty}$ functions.

Let $\mathscr{S} \subseteq \mathscr{P}$ and let $q$ be a point in the natural closure of $\mathscr{S}$ in $\mathscr{Q}$. Then there exists $\left\{q^{n}\right\} \subseteq \mathscr{S}$ such that $q^{n} \rightarrow q$ in the natural topology. Hence, by (4.1) and Lemma 5.1,

$$
\begin{aligned}
\lim _{n} \operatorname{Tr}\left(T^{g^{n}}\right) & =\lim _{n} \int f(g) \theta_{q^{n}}(g) d g \\
& =\int f(g) \theta_{q}(g) d g, \quad f \in \mathfrak{B} .
\end{aligned}
$$

By (4.1), Lemma 6.1 and the definition of associated elements, this says

$$
\lim _{n} \operatorname{Tr}\left(T_{f}^{q^{n}}\right)=\sum_{q^{\prime}} \operatorname{Tr}\left(T_{f}^{q^{\prime}}\right), \quad f \in \mathfrak{B},
$$

where $q^{\prime}$ runs over all elements of $\mathscr{P} \cup \mathscr{R}$ which are associated with $q$. From Lemma 
2.1 , we conclude that every $q^{\prime}$ associated with $q$ is in the hull-kernel closure of $\mathscr{S}$ in $\hat{G}$.

Conversely, let $p \in \hat{G}$ be in the hull-kernel closure of $\mathscr{S}$. Choose $\left\{p^{i}\right\} \subseteq \mathscr{S}$ such that $p^{i} \rightarrow p$ in the hull-kernel topology. Then no subnet $\left\{q^{j}\right\}$ of $\left\{p^{i}\right\}$ can converge in the natural topology to the point at infinity of $\mathscr{2}$. For if it did, Lemma $5.2 \Rightarrow$

$$
\int f(g) \theta_{q^{3}}(g) d g \rightarrow 0, \quad f \in \mathfrak{B} .
$$

By Lemma 2.1 then, $\left\{q^{j}\right\}$ converges hull-kernelwise to no limit at all. This contradicts the fact that $q^{j} \rightarrow p$ in the hull-kernel topology.

Hence all natural cluster points of $\left\{p^{i}\right\}$ lie in the finite part of $\mathscr{Q}$. Let $q$ be such a cluster point and let $\left\{q^{j}\right\}$ be a subnet of $\left\{p^{i}\right\}$ which converges naturally to $q$. By Lemma 5.1

$$
\int f(g) \theta_{q^{\prime}}(g) d g \rightarrow \int f(g) \theta_{q}(g) d g, \quad f \in \mathfrak{B} .
$$

It follows as in the first part that

$$
\lim _{j} \operatorname{Tr}\left(T_{f}^{q^{j}}\right)=\sum_{q^{\prime}} \operatorname{Tr}\left(T_{f}^{q^{\prime}}\right), \quad f \in \mathfrak{B},
$$

where $q^{\prime}$ runs over all elements of $\mathscr{P} \cup \mathscr{R}$ associated with $q$. We conclude that the only possible hull-kernel limits of $\left\{q^{j}\right\}$ must be points which are associated with $q$. The same therefore must be true of $\left\{p^{i}\right\}$; in particular, $p$ is associated with $q$. Q.E.D.

We obtain immediately the

COROLLARY. The hull-kernel topology of $\mathscr{P}$ coincides with the natural topology.

RemarKs. (1) Note we have not determined the complete topology of $\mathscr{P} \cup \mathscr{R}$ since we have not computed the closure of subsets in $\mathscr{R}$. We shall do that for split-rank 1 groups in $\S 8$.

(2) If it is true that all the representations of the principal series are irreducible when $G$ is complex, then (in that case) $\mathscr{R}=\varnothing$ and $\mathscr{P}=\mathscr{Q}$ is closed in $\hat{G}$. It would follow from Harish-Chandra's computation of the Plancherel measure (in [4d, §5]) that $\hat{G}_{r}=\mathscr{P}$. Then from the above corollary, we would get that $\hat{G}_{r}$ is Hausdorff( $\left(^{2}\right)$. Actually, there is evidence to indicate that these facts are true for any semisimple group which contains only one conjugacy class of Cartan subgroups.

(3) Consider the subset $\mathscr{P}_{1}$ of $\mathscr{Q}$ corresponding to the class one principal series

$$
\mathscr{P}_{1}=\{q=W \cdot(\nu, 1): 1 \in \hat{M}, \text { the trivial representation }\} .
$$

Kostant has shown recently [5] that every $T_{v, 1}$ is actually irreducible, that is, $\mathscr{P}_{1} \subseteq \mathscr{P}$. It follows that the hull-kernel topology on $\mathscr{P}_{1}$ is also the natural topology.

7. The discrete series. In this section we shall show that $\hat{G}$ induces the discrete topology on the subset of all square-integrable representations. 
LEMMA 7.1. The points of $\hat{G}$ form closed sets.

Proof. By [3, p. 378], we need only show that for any $T \in \hat{G}=C^{*}(G)^{\wedge}$ and any $f \in C^{*}(G), T_{f}$ is a compact operator. Choose $f_{n} \in C_{0}^{\infty}(G), f_{n} \rightarrow f$ in $C^{*}(G)$. But then

$$
\left\|T_{f}-T_{f_{n}}\right\|_{\infty}=\left\|T_{f-f_{n}}\right\|_{\infty} \leqq\left\|f-f_{n}\right\|_{C^{*}(G)} \rightarrow 0 ;
$$

that is, $T_{f_{n}}$ converges to $T_{f}$ in the uniform operator norm. By [4c, §5], each $T_{f_{n}}$ is a trace class operator. But the trace class operators are compact and the compact operators form a closed subset of all bounded operators in the uniform norm. It follows that $T_{f}$ is a compact operator. Q.E.D.

Now suppose $G$ has a compact Cartan subgroup $B \subseteq K$. By [4j, Theorem 13], $\hat{G}$ contains square-integrable representations if and only if such a $B$ exists. Let $\mathfrak{b}=$ the Lie algebra of $B$. As usual, $W(\mathfrak{g}, \mathfrak{b})$ denotes the Weyl group and $W_{B}$ the subgroup of $W(\mathfrak{g}, \mathfrak{b})$ given by $W_{B}=N(B) / B$. Let $\mathscr{F}=$ the collection of all linear functionals $\lambda: \mathfrak{b} \rightarrow i \boldsymbol{R}$. Call $\lambda$ regular if $\lambda$ is not fixed by any nontrivial member of $W(\mathfrak{g}, \mathfrak{b})$. The character group $\hat{B}$ may be identified with a lattice $\mathscr{L}$ in $\mathscr{F}$ which is invariant under $W_{B}$. Let $\mathscr{L}^{\prime}$ be the regular elements in $\mathscr{L}$, and let $\mathscr{D}=W_{B} \mid \mathscr{L}^{\prime}$, i.e. the orbit space of $\mathscr{L}^{\prime}$ under $W_{B}$.

It follows from [4j, Theorem 16] that $\mathscr{D}$ is embedded in $\hat{G}$ and that $\mathscr{D}$ consists precisely of the square-integrable members of $\hat{G}$.

THEOREM 7.2. The hull-kernel topology on $\mathscr{D}$ is the discrete topology.

For the proof we need a result analogous to Lemma 5.2.

Lemma 7.3. For any $\lambda \in \mathscr{L}^{\prime}$, let $\theta_{\lambda}$ be the invariant eigendistribution of $G$ constructed by Harish-Chandra in [4h, Theorem 3]. Then for any $f \in C_{0}^{\infty}(G)$, we have $\theta_{\lambda}(f) \rightarrow 0$ as $\lambda \rightarrow \infty$ in the discrete topology of $\mathscr{D}$.

Proof. Harish-Chandra proves this result in [4h, Lemma 68]. In fact, he shows that $\theta_{\lambda}(f)$ is rapidly decreasing as a function of $\lambda \in \mathscr{L}^{\prime}$.

REMARK. It seems plausible that Lemma 5.2 might be proven in analogy with [4h, Lemma 68], i.e. by employing the Casimir operator on $G$. Unfortunately, we have not been able to work out all the details.

Proof of Theorem 7.2. Let $\mathscr{S} \subseteq \mathscr{D}$ be an arbitrary subset. It suffices to show that $\mathscr{S}$ is closed in $\hat{G}$. Let $p \in \hat{G}$ be in the hull-kernel closure of $\mathscr{S}$. Choose a net $\left\{p^{i}\right\} \subseteq \mathscr{S}, p^{i} \rightarrow p$ hull-kernelwise. Then no subnet $\left\{q^{j}\right\}$ of $\left\{p^{i}\right\}$ can converge to the point at infinity of $\mathscr{D}$ in the discrete topology. Indeed, suppose $q^{j} \rightarrow \infty$ in the discrete topology. Let $T^{j}$ be any square-integrable representation in the class $q^{j}=W_{B} \cdot \lambda_{j}$. Then, by $[4 \mathrm{j}$, Theorem 16$]$ and Lemma 7.3 , we have

$$
\left|\operatorname{Tr}\left(T_{f}^{\jmath}\right)\right|=\left|\theta_{\lambda_{f}}(f)\right| \rightarrow 0, \quad f \in C_{0}^{\infty}(G) .
$$

Hence by Lemma $2.1,\left\{q^{\dagger}\right\}$ converges hull-kernelwise to no limit. This contradicts the fact that $q^{j} \rightarrow p$ in the hull-kernel topology. 
Thus all discrete cluster points of $\left\{p^{i}\right\}$ lie in the finite part of $\mathscr{D}$. Let $p_{1} \in \mathscr{D}$ be one of them. Then there exists a subnet $\left\{q^{j}\right\}$ of $\left\{p^{i}\right\}$ which converges to $p_{1}$ in the discrete topology, that is $q^{j}=p_{1}$ for large $j$. Hence $p_{1} \in \mathscr{S}$. But $q^{j} \rightarrow p$ in the hull-kernel topology; therefore $p$ is in the hull-kernel closure of $\left\{p_{1}\right\}$. By Lemma 7.1, $p=p_{1} \in \mathscr{S}$.

8. The split-rank one case. In this section we assume $\operatorname{dim} \mathfrak{h}_{+}=1$. We shall also suppose in this final section that $G$ is a matrix group. Then we can find $G_{c}$, a connected semisimple Lie group with Lie algebra $\mathfrak{g}_{c}$ such that $G \subseteq G_{c}$. In this case the subgroup $\Gamma$ of Lemma 3.1 may be taken to be the abelian group

$$
\Gamma=K \cap \exp \sqrt{ }(-1) \mathfrak{h}_{+}
$$

(compare the group $Z_{A}$ in [4i, p. 121]). It follows easily that if $\delta \in \hat{M}$, then $\left.\delta\right|_{M^{\circ}} \in\left(M^{\circ}\right)^{\wedge}$.

We have to deal now with two possibilities: either $A$ is the unique Cartan subgroup up to conjugacy $(\mathscr{D}=\varnothing)$ or there also exists a compact Cartan subgroup $B$ $(\mathscr{D} \neq \varnothing)$. In the latter case, $\mathscr{P} \cup \mathscr{R} \cup \mathscr{D} \subseteq \hat{G}$ and we must prove

LEMMA 8.1. $(\mathscr{P} \cup \mathscr{R}) \cap \mathscr{D}=\varnothing$.

Proof. Let $\mathfrak{u}=$ the universal enveloping algebra of $\mathfrak{g}_{c}$ and let $\mathbb{Z}=$ Cent $\mathfrak{u}$. For any $T \in \hat{G}$, it is known (from [4c, §5]) that $\theta_{T}: f \rightarrow \operatorname{Tr}\left(\int_{G} f(g) T_{g} d g\right), f \in C_{0}^{\infty}(G)$, is a distribution on $G$. It is in fact an eigendistribution of $\mathbf{3}$, i.e. $z \theta_{T}=\chi_{T}(z) \theta_{T}$ where $\chi_{T}: B \rightarrow C$ is a homomorphism. $\chi_{T}$ is called the infinitesimal character of $T$. But the representations in $\mathscr{Q}$ have infinitesimal characters in the preceding sense (whether they are in $\mathscr{P}$ or not). Moreover, if $T \in \mathscr{Q}$ decomposes into $T=\oplus T^{j}, T^{j} \in \mathscr{R}$, it is easy to see that $\chi_{T}=\chi_{T}$, for any $j$. Therefore it is enough to show that no infinitesimal character of any representation in $\mathscr{Q}$ coincides with an infinitesimal character of any representation in $\mathscr{D}$.

We briefly recall a method for obtaining homomorphisms of 3 . Let $a$ be any Cartan subalgebra of a semisimple Lie algebra $g$. Let $\gamma_{\mathfrak{a}}: \mathbb{B} \rightarrow I\left(\mathfrak{a}_{c}\right)$ be the canonical isomorphism of 3 onto $I\left(a_{c}\right)=$ the $W(\mathfrak{g}, \mathfrak{a})$-invariant polynomial functions on $\mathfrak{a}_{c}^{* \cdot}$ For $\lambda \in \mathfrak{a}_{c}^{*}$, let $\chi_{\lambda}^{\mathfrak{a}}: 3 \rightarrow C$ be defined by $\chi_{\lambda}^{\mathfrak{a}}(z)=\gamma_{\mathfrak{a}}(z)(\lambda)$. All homomorphisms of 8 into $C$ are obtained in this way and $\chi_{\lambda}^{\mathfrak{a}}=\chi_{\mu}^{\mathfrak{a}} \Leftrightarrow s \lambda=\mu$ for some $s \in W(\mathfrak{g}, \mathfrak{a})$. (See [4g, $\$ 12]$ and [4h, $\$ 12,19]$ for this material.)

The infinitesimal characters of the representations in $\mathscr{D}$ are computed in [4h, $\S 19]$. Let $T \in \mathscr{D}$, and let $\lambda \in \mathscr{L}^{\prime}$ be a linear functional on $\mathfrak{b}$ determined by the class $T=W_{B} \cdot \lambda$. Extend $\lambda$ to $\mathfrak{b}_{c}$ by complexification. Then $\chi_{T}=\chi_{\lambda}^{\mathfrak{b}}$. The infinitesimal characters of the representations in $\mathscr{Q}$ are computed in [4b, $\$ 4]$. Let $q=W \cdot(\nu, \delta) \in \mathscr{Q}$ and let $\Lambda$ be the highest weight on $\mathfrak{h}_{-}$corresponding to $\left.\delta\right|_{M^{\circ}}$. We define a linear function $\mu$ on $\mathfrak{h}$ by requiring that $\left.\mu\right|_{\mathfrak{h}_{+}}=i v$ and $\left.\mu\right|_{\mathfrak{h}_{-}}=\Lambda+\rho$, where $2 \rho=$ the sum of the positive roots of $\left(\mathfrak{m}, \mathfrak{h}_{-}\right)$. Extend $\mu$ to $\mathfrak{h}_{c}$ by complexification. The infinitesimal character of $T_{v, \delta}$ is exactly $\chi_{\mu}^{\mathfrak{h}}$.

Suppose now that for some $(\nu, \delta) \in \hat{A}_{+} \times \hat{M}$ and some $\lambda \in \mathscr{L}^{\prime}$, we have $\chi_{\lambda}^{\mathfrak{b}}=\chi_{\mu}^{\mathfrak{h}}$. We shall derive a contradiction by showing that $\lambda$ is not regular. We use the results 
of [4i, §24]. Choose $y \in G_{c}$ as in [4i, p. 121]. Then $\mathfrak{b}_{c}^{y}=\mathfrak{h}_{c}$ and by [4h, §12], we have $\chi_{\mu}^{\mathfrak{h}}=\chi_{\mu_{1}}^{\mathfrak{b}}$ where $\mu_{1}(H)=\mu(\operatorname{Ad} y(H)), H \in \mathfrak{b}_{c}$. It follows that there exists $s \in W(\mathfrak{g}, \mathfrak{b})$ so that $s \lambda=\mu_{1}$.

It is possible to choose an ordering on the roots of $(\mathfrak{g}, \mathfrak{h})$ and a positive root $\alpha \in P$ so that if $H_{\alpha} \in \mathfrak{h}_{c}$ satisfies $\alpha(H)=\left(H, H_{\alpha}\right)$ for all $H \in \mathfrak{h}_{c}$, then $H_{\alpha} \in \mathfrak{h}_{+}$. Here $(\cdot, \cdot)$ denotes the Killing form on $\mathfrak{g}_{c}$. If we let $Q=P^{y^{-1}}=\left\{\gamma^{y^{-1}}: \gamma \in P\right\}$, then we may take $Q$ to be the set of positive roots of $(\mathfrak{g}, \mathfrak{b})$. Moreover, it is clear from [4i, $\S 24]$ that $\operatorname{Ad} y^{-1}\left(\mathfrak{h}_{-}\right)=\mathfrak{h}_{-}$and $\operatorname{Ad} y^{-1}\left(\mathfrak{h}_{+}\right) \subseteq i \mathfrak{b}$. But $\lambda \in \mathscr{L}^{\prime}$ implies $\lambda$ takes purely imaginary values on $\mathfrak{b}$. Since $s \lambda \in \mathscr{F}$, the same is true of $s \lambda$. But

and

$$
\mu_{1}\left(\operatorname{Ad} y^{-1} \mathfrak{h}_{+}\right)=\mu\left(\mathfrak{h}_{+}\right)=i \nu\left(\mathfrak{h}_{+}\right) \subseteq i R
$$

$$
\mu_{1}\left(\operatorname{Ad} y^{-1} \mathfrak{h}_{+}\right)=s \lambda\left(\operatorname{Ad} y^{-1} \mathfrak{h}_{+}\right) \subseteq s \lambda(i \mathfrak{b}) \subseteq R
$$

Therefore $\nu=0$.

Let $\beta=\alpha^{y^{-1}} \in Q$ and let $H_{\beta} \in \mathfrak{b}_{c}$ be such that $\beta(H)=\left(H, H_{\beta}\right), H \in \mathfrak{b}_{c}$. Then $s \lambda\left(H_{\beta}\right)=\mu_{1}\left(H_{\beta}\right)=\mu\left(H_{\beta}^{y}\right)=\mu\left(H_{\alpha}\right)=i v\left(H_{\alpha}\right)=0$. It follows that $s \lambda$ is not regular, and therefore $\lambda$ itself is not regular. This contradiction completes the proof.

We can now give the complete topology of $\hat{G}_{r}$.

THEOREM 8.2. (1) Let $A$ be the unique Cartan subgroup of $G$. Then $\hat{G}_{r}=\mathscr{P} \cup \mathscr{R}$ and the dual topology of $\hat{G}_{r}$ is determined as follows:

(a) the closure of a set $\mathscr{S} \subseteq \mathscr{P}$ is as in Theorem 6.2;

(b) the topology of $\mathscr{R}$ is discrete.

(2) Suppose $G$ also has a compact Cartan subgroup. Then $\hat{G}_{r}=\mathscr{P} \cup \mathscr{R} \cup \mathscr{D}$ is a disjoint union, $\mathscr{P} \cup \mathscr{R}$ is open in $\hat{G}_{r}, \mathscr{D}$ is open in $\hat{G}_{r}$, and we have

(a) the topology of $\mathscr{P} \cup \mathscr{R}$ is as in part (1);

(b) the topology of $\mathscr{D}$ is discrete.

Proof. We first show that the dual topology on $\mathscr{R}$ is discrete. When $\operatorname{dim} \mathfrak{h}_{+}=1$, the group $W$ has precisely two elements and the only $\nu \in \mathfrak{h}_{+}^{*}$ fixed by both of them is $\boldsymbol{\nu}=\mathbf{0}$. Hence $\mathscr{Q}-\mathscr{P} \subseteq\{\boldsymbol{W} \cdot(0, \delta): \delta \in \hat{M}\}$, a discrete, countable-and therefore closed-subset of $\mathscr{Q}$ in the natural topology.

If $\mathscr{Q}-\mathscr{P}$ is empty, then $\mathscr{R}=\varnothing$ and there is nothing to prove. Otherwise, let $\mathscr{S} \subseteq \mathscr{R}$ be an arbitrary subset. We show it is closed in $\hat{G}$. Let $p \in \hat{G}$ be in the hullkernel closure of $\mathscr{S}$. Choose a net $\left\{p^{i}\right\} \subseteq \mathscr{S}, p^{i} \rightarrow p$ hull-kernelwise. Then no subnet $\left\{q^{j}\right\}$ of $\left\{p^{i}\right\}$ can converge in the discrete topology to the point at infinity of $\mathscr{R}$. Indeed, suppose $q^{j} \rightarrow \infty$ in the discrete topology. By the remark at the beginning of $\S 6$, there exists a unique element $\pi^{j} \in \mathscr{Q}-\mathscr{P}$ such that $q^{j}$ is a direct summand of $\pi^{j}$. Claim: $\pi^{j} \rightarrow \infty$ in the natural topology of $\mathscr{Q}$.

To prove this, let $\mathscr{C}$ be a compact subset of $\mathscr{Q}$ (in the natural topology). Then $\mathscr{C} \cap(\mathscr{Q}-\mathscr{P})$ is a finite set. Let $\mathscr{R}_{\mathscr{C}}=$ those elements of $\mathscr{R}$ which arise as direct summands of $\mathscr{C} \cap(\mathscr{Q}-\mathscr{P})$. Then $\mathscr{R}_{\mathscr{C}}$ is finite. Hence $q^{j} \notin \mathscr{R}_{\mathscr{C}}$ for all large $j$. Therefore $\pi^{j} \notin \mathscr{C}$ for all large $j$; that is, $\pi^{j} \rightarrow \infty$ in the natural topology of $\mathscr{Q}$. 
By Lemma 5.2, we get

$$
\operatorname{Tr}\left(\pi_{f}^{j}\right)=\int f(g) \theta_{\pi^{j}}(g) d g \rightarrow 0, \quad f \in C_{0}^{\infty}(G) .
$$

It follows from [3, Corollary 1, p. 390] that

$$
\left\|\pi_{f}^{j}\right\|_{\infty} \rightarrow 0, \quad f \in C^{*}(G) .
$$

But $q^{j}$ is a direct summand of $\pi^{j}$ and so

$$
\left\|q_{f}^{j}\right\|_{\infty} \rightarrow 0, \quad f \in C^{*}(G) .
$$

Hence by [3, Corollary 1, p. 382], no subnet of $\left\{q^{\}}\right\}$can converge hull-kernelwise to any limit. This contradicts $p^{i} \rightarrow p$ in the hull-kernel topology. Hence no subnet of $\left\{p^{i}\right\}$ can converge discretely to $\infty$.

The proof is completed now as in Theorem 7.2. Choose a discrete cluster point $p_{1}$ of $\left\{p^{i}\right\}$ in $\mathscr{R}$ and a subnet $\left\{q^{j}\right\}$ of $\left\{p^{i}\right\}$ which converges to it discretely. It follows that $p_{1} \in \mathscr{S}$ and $p$ is in the hull-kernel closure of $\left\{p_{1}\right\}$. By Lemma 7.1, $p=p_{1} \in \mathscr{S}$.

Now $\mathscr{P} \cup \mathscr{R}$ is the hull-kernel closure of $\mathscr{P}$, so $\mathscr{P} \cup \mathscr{R}$ is always closed in $\hat{G}$. In the second case, $\mathscr{D}$ is closed by the proof of Theorem 7.2. In fact, by Theorems 6.2 and 7.2, we are done once we observe that: in case (1), $\mathscr{P} \cup \mathscr{R}$ is the smallest closed subset of $\hat{G}$ which supports the Plancherel measure (see [4e, $\S 1]$ ); and in case (2), $\mathscr{P} \cup \mathscr{R} \cup \mathscr{D}$ is the smallest closed subset of $\hat{G}$ supporting the Plancherel measure (see [4i, Lemma 56]).

RemarKs. (1) It is possible for $\#(\mathscr{R})=\infty$. In [7, p. 421] Takahashi shows that for the universal covering group of $S O_{e}(4,1)$, we have $\#(\mathscr{Q}-\mathscr{P})=\infty$. By the remark at the beginning of $\S 6$, it follows that $\#(\mathscr{R})=\infty$ also.

(2) It is well known that $\mathscr{D}$ is not open in general in $\hat{G}$. For example when $G=S L(2, R)$, a particular member of the discrete series can be obtained as a limit point of the complementary series. Dixmier has conjectured that the points of $\mathscr{D}$ are open in $\hat{G}_{r}$ (see $\left.[2,18.9 .1 \mathrm{c}]\right)$. We have verified this fact in the case of splitrank one semisimple groups.

\section{REFERENCES}

1. F. Bruhat, Sur les représentations induites des groupes de Lie, Bull. Soc. Math. France 84 (1956), 97-205. MR 18, 907.

2. J. Dixmier, Les $C^{*}$-algèbres et leurs représentations, Cahiers Scientifiques, fasc. 29, Gauthier-Villars, Paris, 1964. MR 30 \#1404.

3. J. M. G. Fell, The dual spaces of $C^{*}$-algebras, Trans. Amer. Math. Soc. 94 (1960), 365-403. MR $26 \# 4201$.

4. Harish-Chandra, a) Representations of a semisimple Lie group on a Banach space. I, Trans. Amer. Math. Soc. 75 (1953), 185-243. MR 15, 100.

b) Representations of semisimple Lie groups. II, Trans. Amer. Math. Soc. 76 (1954), 26-65. MR 15, 398.

c) Representations of semisimple Lie groups. III, Trans. Amer. Math. Soc. 76 (1954), 234-253. MR 16, 11. 
d) The Plancherel formula for complex semisimple Lie groups, Trans. Amer. Math. Soc. 76 (1954), 485-528. MR 16, 111.

e) A formula for semisimple Lie groups, Amer. J. Math. 79 (1957), 733-760. MR 20 \#2633.

f) Some results on an invariant integral on a semisimple Lie algebra, Ann. of Math. (2) 80 (1964), 551-593. MR 31 \#4862b.

g) Invariant eigendistributions on a semisimple Lie group, Trans. Amer. Math. Soc. 119 (1965), 457-508. MR 31 \#4862d.

h) Discrete series for semisimple Lie groups. I: Construction of invariant eigendistributions, Acta Math. 113 (1965), 241-318. MR 36\#2744.

i) Two theorems on semi-simple Lie groups, Ann. of Math. (2) 83 (1966), 74-128. MR 33 \#2766.

j) Discrete series for semisimple Lie groups. II: Explicit determination of the characters, Acta Math. 116 (1966), 1-111. MR 36 \#2745.

5. B. Kostant, On the existence and irreducibility of certain series of representations, Bull. Amer. Math. Soc. 75 (1969), 627-642.

6. I. Satake, On representations and compactifications of symmetric Riemannian spaces, Ann. of Math. (2) 71 (1960), 77-110. MR 22 \#9546.

7. R. Takahashi, Sur les représentations unitaires des groupes de Lorentz généralisés, Bull. Soc. Math. France 91 (1963), 289-433. MR 31 \#3544.

UNIVERSITY OF MARYLAND,

College Park, Maryland 20742 